JUSTYNA WesOla

ORCID: 0000-0003-1098-7171

Université de Wrocław

justyna.wesola@uwr.edu.pl

\title{
TRANSFERT DE LITTÉRATURE ÉTRANGÈRE ET BILINGUISME. CAS DE LA LITTÉRATURE POLONAISE DANS LES LANGUES PÉRIPHÉRIQUES D'ESPAGNE ET DE SES TRADUCTIONS PRÉALABLES EN CASTILLAN
}

\section{INTRODUCTION}

Comme l'a observé Marcin Kurek, « la littérature polonaise passe toujours les Pyrénées par la porte française $»^{1}$. Et à l'appui de cette thèse, il a présenté les dates de publication de la poésie de Tadeusz Różewicz et d'Adam Zagajewski, qui, effectivement, semblent confirmer le fait. Cette idée de Kurek a été l'une des motivations qui m'ont amenée à m'intéresser à la question : l'entrée de la littérature polonaise en Espagne est-elle conditionnée de la sorte, ou, pour le dire autrement, doit-elle toujours être traduite préalablement en une langue « centrale » pour entrer ensuite au pays de Cervantes dans d'autres traductions analogues?

Une réponse exhaustive à cette question réclamerait une analyse documentée et approfondie dépassant le cadre d'un article comme celui-ci. Selon les données recueillies (essentiellement dans les catalogues de la Bibliothèque nationale polonaise $^{2}$ et la Base de datos de libros editados en España du Ministère de l'Éduca-

${ }^{1}$ M. Kurek, « Przypisy tłumacza w hiszpańskich przekładach współczesnej literatury polskiej » [Les notes du traducteur dans les traductions de littérature polonaise contemporaine], [dans :] E. Skibińska (dir.), Przypisy tlumacza, Księgarnia Akademicka, Wrocław-Kraków 2009, pp. 82-83.

2 Biblioteka Narodowa, <https://bn.org.pl/> [consulté le 23.04.2020]. 
tion, de la Culture et des Sports espagnol $-\mathrm{MECD}^{3}$ ), le nombre de livres entrant dans la catégorie « littérature polonaise » édités en Espagne dépassait le millier en $2015^{4}$ et avoisine actuellement les $1200^{5}$. Je me risquerai toutefois à proposer un premier aperçu de la question.

L'édition de littérature polonaise en Espagne pourrait s'apparenter à un transfert entre deux langues de statut équivalent, que Johan Heilbron appellerait semi-périphériques ${ }^{6}$. En effet, il ne faut pas oublier que dans ce pays, à côté du castillan qui est langue officielle sur tout le territoire, il existe aussi d'autres langues qui, depuis la fin du régime du général Francisco Franco, se portent de mieux en mieux. Ce sont le catalan, le galicien, le basque, l'aragonais, le léonais et l'aranais, que je cite dans l'ordre en fonction de leur nombre de locuteurs et de l'ancienneté de leur tradition littéraire. Le catalan est en effet le plus parlé (environ 10 millions de locuteurs ${ }^{7}$ ) et la naissance de sa littérature est datée de la fin du $\mathrm{XI}^{\mathrm{e}}$, début du XII ${ }^{\mathrm{e}}$ siècle. Le galicien vient en seconde position avec 3 millions de locuteurs et une littérature qui date également du moyen-âge (époque à laquelle elle est encore indifférenciée de la littérature portugaise), et le basque (euskera/euskara), en troisième position avec une littérature dont la forme écrite est plus jeune, puisqu'elle date d'environ 500 ans, et environ un million de locuteurs. Il faut cependant préciser que la moitié de ces derniers n'ont qu'une connaissance incomplète, souvent passive de la langue ${ }^{8}$. Quant aux trois dernières, leur importance est marginale. L'aragonais et le léonais appartiennent de fait à l'aire dialectale de l'espagnol et comptent respectivement 125000 et 25000 locuteurs, et l'aranais est un dialecte gascon-occitan parlé par 10000 personnes.

${ }^{3}$ Base de datos de libros editados en España, < http://www.culturaydeporte.gob.es/cultura/ libro/bases-de-datos-del-isbn/base-de-datos-de-libros.html > [consulté le 23.04.2020].

${ }^{4}$ Pour plus de détails à propos de la littérature polonaise en Espagne avant cette date, voir : J. Wesoła, «La littérature polonaise en Espagne dans les années 1989-2014 », [dans :] E. Skibińska, R. Solová, K. Gostkowska (dir.), Vingt cinq ans après... Traduire dans une Europe en reconfiguration, Orizons, Paris 2015, pp. 105-124.

5 Il est difficile de donner un chiffre exact car la définition de la « littérature polonaise » est question d'interprétation (l'expression peut se référer aux œuvres écrites en polonais, aux œuvres d'auteurs d'origine polonaise, aux seules « belles lettres » ou, au contraire, à un ensemble d'écrits plus vaste). En outre, les bases de données ne sont pas forcément complètes ou certains ouvrages peuvent y être classés comme appartenant à une autre langue. Elle peuvent également omettre les traductions indirectes, qui n'ont pas été réalisées à partir de l'original.

6 J. Heilbron, « Towards a Sociology of Translation », European Journal of Social Theory 2(4), 1999, pp. 433-435, [cité d'après :] E. Skibińska, Kuchnia tlumacza [La cuisine du traducteur], Universitas, Kraków 2008, pp. 36-37.

${ }^{7}$ Nombres de locuteurs selon les données de : «Idiomas de España », [dans :] Wikipedia. La enciclopedia libre, $<$ https://es.wikipedia.org/wiki/Idiomas_de_Espa\% $\%$ C3\%B1a\#Resumen_de_todas_las_lenguas> [consulté le 8.05.2020].

8 «Euskera», [dans :] Wikipedia. La enciclopedia libre, $<$ https://es.wikipedia.org/wiki/Euskera \#N\%C3\%BAmero_de_hablantes> [consulté le 8.05.2020]. 
Dans leur immense majorité, les locuteurs des langues minoritaires sont bilingues et lisent sans problème la littérature traduite en espagnol. Des dizaines d'œuvres de la littérature polonaise ont cependant été traduites dans leurs langues périphériques. Cette situation soulève un ensemble de questions : comment fonctionne le transfert de littérature étrangère lorsque le lectorat est bilingue ? L'existence d'une traduction dans la langue dominante influence-t-elle l'édition d'une traduction en langue dominée ? Est-elle une consécration, une motivation à traduire le texte dans la langue minoritaire, ou au contraire, rend-elle cette traduction inutile puisque la langue dominante permet déjà au lecteur de connaître l'œuvre?

\section{PANORAMA DES TRADUCTIONS DE L'ESPAGNE PÉRIPHÉRIQUE}

Selon la typologie de Pascale Casanova, le polonais est une langue dominée, tout comme les langues périphériques d'Espagne, et les traductions entre elles relèvent du « cas très rare $»^{9}$. On est cependant en droit de penser qu'il n'entre pas vraiment dans la même catégorie, vu sa $24^{\mathrm{e}}$ position dans le classement mondial des langues par nombre de locuteurs (selon les données d'Ethnologue de 2013) ${ }^{10}$ et ses cinq prix Nobel de littérature. L'importance marginale des langues périphériques d'Espagne transparaît ne serait-ce que dans le fait que, le catalan mis à part, elles ne figurent pas dans le catalogue de la Bibliothèque nationale polonaise (lequel distingue tout de même 81 langues dont certaines sont plus exotiques et plus éloignées géographiquement). Certains titres publiés dans ces langues y sont par erreur classés dans le domaine espagnol.

Selon la base de données du MECD, les Catalans disposent aujourd'hui de plus de 80 ouvrages de littérature polonaise traduits dans leur langue, les Galiciens, sept, et les Basques, trois ${ }^{11}$. Les usagers des autres langues régionales n'en ont aucun. Pour le léonais, langue très proche du castillan reprise dans la base de données sous son nom synonyme d'asturiano, le MECD signale au total 2365 traductions, dont 97 de l'espagnol, 66 de l'anglais, 41 du français, 15 du catalan, 10 de l'allemand, et aucune du polonais. Pour l'aragonais (512 entrées dans la

9 P. Casanova, « Consécration et accumulation de capital littéraire. La traduction comme échange inégal », Actes de la Recherche en Sciences Sociales 144, 2002, pp. 9-10.

${ }^{10}$ Ethnologue. Languages of the World, <https://www.ethnologue.com/> [consultéle 27.01.2013].

11 Au moment où nous terminions cet article, en 2020, les éditions Elkar ont sorti Erabili goldea hilen hezurren gainetik, version basque de Sur les ossements des morts d'Olga Tokarczuk, dans une traduction d'Amaia Apalauza. L'Euskal Itzulpengintzaren datu-basea "Nor da Nor" [Base de données de traductions basques Who is who] signale aussi des textes des auteurs suivants publiés dans des revues ou en ligne : Henryk Sienkiewicz (1957), Czesław Miłosz (1984, 1985), Witold Gombrowicz (1987), Leopold Staff (1991), Wisława Szymborska (1997, 2001, 2004, 2011, 2012), Stanisław Lem (2006), Jacek Dehnel (2007), Sławomir Mrożek (2013), Maciej Wojtyszko (2013), Stanisław Jerzy Lec (2016). 
base de données) et l'aranais (217), il s'agit surtout de littérature originale, de manuels scolaires, de livres sur la région et d'ouvrages linguistiques consacrés à ces langues. Les rares traductions sont surtout à partir du catalan (vraisemblablement en raison de la proximité géographique), ou il s'agit de littérature pour enfants (le MECD signale des traductions et adaptations des Aventures de Tom Sawyer, de Pinocchio et de Heidi).

Les 90 œuvres de littérature polonaise traduites dans les langues minoritaires d'Espagne (toutes langues confondues) peuvent sembler peu de chose face aux plus de mille ouvrages traduits en castillan, mais il ne faut pas oublier que ces langues n'ont cessé d'être interdites dans le domaine public qu'après la mort du général Franco et n'ont été reconnues officiellement par la Constitution qu'en 1978. Rappelons cependant que dans les communautés autonomes qui les parlent, elles ont le statut de langues officielles à côté du castillan.

\section{PREMIÈRE TRADUCTION QUADRUPLE DE LITTÉRATURE POLONAISE. LES EUVRES D'OLGA TOKARCZUK EN ESPAGNE}

L'année 2019 représente un moment historique avec, pour la première fois, la publication en Espagne d'un livre polonais en quatre versions linguistiques : espagnole, catalane, galicienne et basque. Il s'agit de Zgubiona dusza d'Olga Tokarczuk. Les quatre versions ${ }^{12}$ sont parues chez le même éditeur barcelonais, Thule Ediciones. L'original, illustré par Joanna Concejo — une artiste de Słupsk qui vit actuellement en France et porte le nom espagnol de son mari - a été primé en mars 2018, dans la catégorie FICTION ${ }^{13}$, à l'occasion du Bologna Ragazzi Award, prix international prestigieux décerné chaque année pendant la Foire du livre de jeunesse de Bologne. Ce prix ne semble cependant pas avoir directement influencé la décision de l'éditeur, pas plus d'ailleurs que le Prix Booker décerné à Tokarczuk la même année ni le grand nombre de traductions précédentes de ses œuvres dans d'autres langues. Voici comment José Díaz, le directeur de Thule Ediciones, parle des circonstances de l'édition du livre :

Nous sommes une petite maison d'édition et normalement, nous n'avons pas les moyens d'entrer en compétition pour les livres primés. Par principe, on ne vise pas les ouvrages primés à Bologne parce qu'il y a toujours un autre éditeur qui peut payer plus que nous.

12 O. Tokarczuk, El alma perdida, trad. X. Farré i Vidal / L'anima perduda, trad. X. Farré i Vidal / A alma perdida, trad. A. Garrido González / Arima galdua, trad. I. García Quintana, Thule Ediciones, Barcelona 2019. Dans les notes, je ne présente que les données bibliographiques des traductions en langues périphériques évoquées dans l'article et des versions espagnoles correspondantes.

13 Rédaction du site Wirtualny Wydawca, « Bologna Ragazzi Award przyznane », 12.02.2018, $<$ https://wirtualnywydawca.pl/2018/02/bologna-ragazzi-award/> [consulté le 8.05.2020]. Je remercie Katarzyna Biernacka-Licznar d'avoir attiré mon attention sur ce fait et de m'avoir mise en contact avec Mme Dorota Hartwich de la maison d'édition Wydawnictwo Format. 
El alma perdida m'a été proposé un an après le prix de Bologne par Karolina Jaszecka, agent de l'éditeur en Espagne, parce qu'aucun autre éditeur espagnol ne s'y était intéressé. C'est en fait un livre un peu particulier, un album illustré pour adultes, pas pour enfants, et il n'y a pas beaucoup d'éditeurs à qui ce genre d'œuvres convienne. Sur ses 52 pages, la plupart ne contiennent que des illustrations, il n'y a pas de texte, c'est pour les lecteurs visuels. Aucun éditeur n'en a voulu, car ils le trouvaient « difficile». Cette singularité en fait aussi un livre difficile du point de vue commercial, parce qu'on n'en vend pas beaucoup, il s'adresse à un public très particulier. Olga n'avait pas beaucoup de titres publiés en espagnol, de sorte que son lectorat n'était pas très développé chez nous.

Karolina a pensé que l'ouvrage correspondait à notre profil - il est effectivement dans notre ligne éditoriale —, et quand elle me l'a présenté, le monde s'est arrêté, j'ai eu la tête qui tourne (le syndrome de Stendhal). Il était d'une beauté remarquable. Il méritait d'être édité, et nous l'avons eu à un prix raisonnable.

La raison pour laquelle nous l'avons édité, malgré des prévisions commerciales incertaines, c'est donc la beauté, ni plus ni moins. C'est un beau livre, voilà la raison de sa publication.

Et le travail de Joanna Concejo y est pour beaucoup, parce que si le texte est formidable, Joanna en a fourni une merveilleuse interprétation esthétique ${ }^{14}$.

Cette longue citation montre bien la complexité du processus au terme duquel Zgubiona dusza a été publié. Le principal critère décisif de sa publication a été la qualité du livre, surtout sur le plan graphique, mais on voit aussi tout l'ensemble de facteurs qui ont influencé la décision : possibilités financières et capacité de concurrence d'une petite maison d'édition, caractère particulier du livre et du public cible, présence antérieure de l'auteure sur le marché éditorial, recommandation d'une personne compétente, profil de l'éditeur. Dans son e-mail, le directeur de Thule nous apporte encore une information d'importance cruciale pour le présent article : au départ, Zgubiona dusza ne devait être publié qu'en espagnol et en catalan (« dans les langues dans lesquelles nous publions habituellement » ${ }^{15}$ ),

14 «Somos una editorial pequeña y normalmente no podemos competir por libros premiados, así que por principio no perseguimos los premiados en Bolonia pues siempre hay otra editorial que puede pagar más que nosotros. El alma perdida me lo presentó la agente de la editorial en España, Karolina Jaszecka, un año después de haber sido premiado en Bolonia y sin que ningún otro editor en español se interesase. Porque es un libro singular, es un álbum ilustrado para adultos, no para niños, así que hay pocas editoriales en las que este tipo de obra puede encajar. De las 52 páginas, la mayoría son solo ilustraciones, sin texto, requiere un lector visual. Ningún otro editor lo quiso, porque lo consideraban "difícil". Esa misma singularidad lo hace un libro complicado comercialmente, pues no se suelen vender mucho, se dirige a un público muy particular. Olga tenía muy pocos títulos publicados en español, así que tampoco tenía una base de lectores amplia. A Karolina le pareció que encajaba con nuestro perfil, pues publicamos libros en esa línea, y cuando me lo presentó se detuvo el mundo, me dio vértigo (síndrome de Stendhal), me pareció un libro bellísimo que merecía editarse y lo conseguimos a un precio asequible. Así que la razón de la publicación, pese a sus malos presagios comerciales, es ni más ni menos que la belleza. Es un libro bello, esa es la razón principal para haberlo publicado. Y en ello la labor de Joanna Concejo es fundamental, porque es un texto extraordinario, pero Joanna hace una interpretación estética maravillosa ». Réponse de l'éditeur par e-mail le 30.04.2020. Mes remerciements à M. José Díaz pour sa gentillesse et son autorisation de la publier. Je remercie également Mme Dorota Hartwich de la maison d'édition Wydawnictwo Format de m'avoir mise en contact avec lui.

15 J. Díaz, e-mail du 30.04.2020. 
mais le prix Nobel de Tokarczuk a décidé l'éditeur à le faire paraître également en galicien et en basque, et à avancer sa publication en novembre 2019 (elle était initialement prévue pour mars 2020).

Comme l'a fait remarquer José Díaz, Tokarczuk était encore mal connue en Espagne à ce moment. Des traductions de Prawiek i inne czasy étaient certes parues simultanément en 2001 en espagnol (Un lugar llamado antaño ${ }^{16}$ ) et en catalan (Un lloc anomenat antany ${ }^{17}$ ), la première, d'Ester Rabasco Macías, et la seconde, d'Anna Rubió i Rodón et Jerzy Sławomirski, tandem réputé de traducteurs de littérature polonaise, mais Olga Tokarczuk avait ensuite été perdue de vue par les éditeurs espagnols. Il avait fallu attendre 2016 pour que la version castillane de Prowadź swój plug przez kości umarlych ${ }^{18}$ paraisse à Madrid.

La raison indirecte de l'édition de cette dernière traduction est un bon exemple de la complexité de la situation linguistique en Espagne et de la grande variété de facteurs qui entrent en compte sur ce marché éditorial. Il ne faut pas oublier que le domaine de l'espagnol ne se limite pas à la Péninsule ibérique, mais englobe aussi les deux Amériques. C'est ainsi que Sobre los huesos de los muertos, la traduction d'Abel Murcia, a commencé par paraître un an plus tôt, en 2015, à Mexico ${ }^{19}$. C'est peut-être sa réception favorable qui a décidé de sa publication également dans la Péninsule. D'autre part, l'édition mexicaine a vraisemblablement été inspirée par le succès du livre en France, où il est paru en 2012 dans une traduction de Margot Carlier rééditée ensuite en 2014 et (après la sortie de l'édition espagnole) en 2017. Le site de vente mexicain Sanborns cite dans sa description un passage de la critique du magazine français $L^{\prime}$ Express :

Olga Tokarczuk propose un fascinant polar aux accents poétiques et fantastiques [...] Sur les ossements des morts passe avec malice de l'intrigue criminelle et zoologique au pamphlet politique sans concession. (L'Express $)^{20}$

L'origine du titre espagnol est du reste sans mystère : le traducteur a luimême expliqué qu'il avait repris la formule du titre français, Sur les ossements des morts $^{21}$. Les traductions anglaises des deux romans (Prawiek... et Prowadź...)

16 O. Tokarczuk, Un lugar llamado antaño, trad. E. Rabasco Macías, Editorial Lumen, Barcelona 2001.

17 O. Tokarczuk, Un lloc anomenat antany, trad. A. Rubió i Rodón, J. Sławomirski, Edicions Proa, Barcelona 2001.

18 O. Tokarczuk, Sobre los huesos de los muertos, trad. A. Murcia, Siruela, Madrid 2016.

19 O. Tokarczuk, Sobre los huesos de los muertos, trad. A. Murcia, Océano, México 2015.

20 « Fascinante novela criminal con toques poéticos y fantásticos... Sobre los huesos de los muertos salta con malicia de la intriga criminal y zoológica al panfleto político que no sabe hacer concesiones » (L'Express, Sólo Sanborns, <https://www.sanborns.com.mx/producto/29463/sobre-loshuesos-de-los-muertos/> [consulté le 08.05.2020]). La citation française originale présentée dans le texte est tirée de : B. Liger, «Le polar zoologique d'Olga Tokarczuk », L'Express, 20.09.2012, <https:// www.lexpress.fr/culture/livre/sur-les-ossements-des-morts_1163716.html> [consulté le 8.05.2020].

21 A. Murcia, «Un thriller metafísico », Instituto de Estudios Turolenses, Revista Turia, <http:// www.ieturolenses.org/revista_turia/index.php/actualidad_turia/cat/lecturas/post/un-triller-metafisico/> [consulté le 8.05.2020]. 
sont postérieures, de même que la mise à l'écran du second (2017). Mais jusqu'en 2019, les 37 autres langues dans lesquelles Tokarczuk a été traduite n'ont pas été un argument de poids suffisant pour qu'aucun de ses livres ne soit traduit en catalan, galicien ou basque. Le 23 octobre 2019, l'éditeur madrilène Anagrama a publié une traduction de Bieguni (Los errantes ${ }^{22}$ ), avec sur la couverture un paratexte ne laissant aucun doute sur ses motivations : «Premio Man Booker International $»^{23}$. La version catalane du livre est sortie le lendemain chez l'éditeur barcelonais Rata et s'intitule $\operatorname{Cos}^{24}$.

\section{AUTRES TRADUCTIONS BASQUES}

À part Zgubiona dusza de Tokarczuk, deux autres titres de deux autres auteurs ont été traduits en basque jusqu'à présent. Panny z Wilka de Jarosław Iwaszkiewicz est paru en 2004 à Guipuscoa sous le titre de Wilkoko andereñoak ${ }^{25}$, dans une traduction d'Adam Zawiszewski. Dix ans plus tard, le traducteur a défendu une thèse de doctorat en linguistique écrite en basque. Il travaille aujourd'hui à l'Université du Pays basque (Euskal Herriko Unibertsitatean). Wilkoko andereñoak a été publié par les éditions Erein eta Igela dans une collection intitulée «Littérature mondiale ». La collection se compose de traductions sélectionnées par le comité de l'Association des traducteurs de langue basque (EIZIE, Euskal Itzultzaile, Zuzentzaile eta Interpreteen Elkartea) qui organise un concours de traduction annuel depuis $1990^{26}$. L'EIZIE publie une liste de textes qu'elle aimerait voir traduits, les concurrents traduisent un extrait qu'ils envoient de façon anonyme, et la traduction de l'ouvrage entier est ensuite confiée au vainqueur ${ }^{27}$. Selon l'information reçue du traducteur, c'est également ainsi que la procédure s'est déroulée l'année de la parution de Panny z Wilka ${ }^{28}$.

L'édition basque a coïncidé avec la parution d'une édition polonaise des Opowiadania d'Iwaszkiewicz à Madrid ${ }^{29}$. Dans le cas de Panny..., il serait difficile de voir un rapport entre la traduction basque et la traduction préalable en

22 O. Tokarczuk, Los errantes, trad. A. Orzeszek-Sujak, Anagrama, Madrid 2019.

23 «Los errantes - Editorial Anagrama », <https://www.anagrama-ed.es/libro/panorama-denarrativas/los-errantes/9788433980533/PN\%201016> [consulté le 23.04.2020].

24 O. Tokarczuk, Cos, trad. X. Farré i Vidal, Rata, Barcelona 2019; Rata Books, <https:// www.facebook.com/RataBooks/photos/a.264243900625544/929163320800262/?type=3\&theater>, 24.10.2019 [consulté le 14.05.2020].

25 J. Iwaszkiewicz, Wilkoko andereñoak, trad. A. Zawiszewski, Alberdania, Guipuscoa 2004.

${ }^{26}$ EIZIE Elkartea, « Bildumako liburuak » [Livres de la collection], <https://eizie.eus/eu/argitalpenak/literatura_unibertsala/orainarte> [consulté le 14.05.2020].

27 EIZIE Elkartea, « Itzulpen-lehiaketa 2019 » [Concours de traduction 2019], <https://eizie. eus/eu/argitalpenak/literatura_unibertsala/lehiaketa2019> [consulté le 14.05.2020].

28 A. Zawiszewski, e-mail du 8.05.2020. Je remercie le traducteur pour les informations qu'il m'a fournies sur les circonstances de l'édition de Wilkoko andereñoak.

29 J. Iwaszkiewicz, Opowiadania, Mediasat Group, Madrid 2004. 
espagnol, car celle-ci a été réalisée 20 ans plus tôt, en $1984^{30}$. Selon les données de la Bibliothèque nationale, Las señoritas de Wilko est parue cette année-là en même temps que El bosque de los abedules, la traduction de Brzezina du même auteur. Le passage par la France pourrait sembler une bonne explication dans ce cas, car les deux œuvres ont aussi été publiées en même temps de l'autre côté des Pyrénées. Seulement... c'était en 1938 (Demoiselles de Wilko, Bois de bouleaux). La réédition française de 1979 — qui s'explique par la nomination aux Oscars de la version cinématographique des Demoiselles... par Wajda — n'a probablement pas non plus inspiré l'édition espagnole, étant donné le long intervalle de cinq ans qui les sépare. Toutefois, le fait est que l'édition française est antérieure. Quant aux rééditions françaises (en 1985, 1992 et 1993) et aux traductions anglaise (2002, mais il y a peut-être eu une traduction antérieure dans une anthologie) et allemande (1985), elles sont postérieures à la version espagnole.

Il y a lieu de noter, en outre, qu'une autre version espagnole de Brzezina est également parue en 1984, de l'autre côté de l'océan, au Mexique. Elle est due aux traducteurs Barbara Stawicka-Pirecka et Mario Muñoz. Mais il ne semble pas qu'il y ait eu de corrélation entre ces deux versions, ni qu'on puisse parler d'une influence hispano-américaine. Pour preuve, Madre Juana de los Ángeles est paru en 1975 (réédité en 1977) à Mexico et en 1982 à La Havane, mais à ce jour, n'a toujours pas été édité en Espagne, alors que les Portugais connaissent depuis longtemps cette œuvre d'Iwaszkiewicz (éditée à Lisbonne en 1962). En 1977, ce dernier était aussi l'un des 22 auteurs polonais dont les nouvelles avaient été traduites par le futur Nobel portugais José Saramágo, probablement à partir du français.

La liste des livres polonais en version basque se termine à ce jour avec Chrystus z karabinem na ramieniu de Ryszard Kapuściński, sorti en 2019 sous le titre de Kristo fusilarekin ${ }^{31}$ dans une traduction d'Amaia Apalauza Ollo, traductrice et linguiste, docteure de la même Alma Mater que Zawiszewski, et tout comme lui, lauréate du concours de l'EIZIE (en 2015) ${ }^{32}$. Elle est depuis 2016 membre sans droit de vote de l'Académie de langue basque (Euskaltzaindia) ${ }^{33}$. Apalauza Ollo a réalisé sa traduction à partir de la version castillane, mais en consultant dans les cas douteux son amie Sonia Kolakzec [sic! - Kołaczek ?], qui connaît le basque. Dans une interview à l'Euskal Irrati Telebista, Apalauza Ollo explique qu'elle a choisi Kapuściński parce que c'est l'une des plus importantes figures du journalisme international et « une référence à de nombreux points de vue », et ce livre,

30 J. Iwaszkiewicz, Las señoritas de Wilko, trad. B. Żaboklicka, J.R. Monreal, Bruguera, Barcelona 1984.

31 R. Kapuściński, Kristo fusilarekin, trad. A. Apalauza Ollo, Katakrak, Iruñea-Pamplona 2019.

32 EIZIE Elkartea, « Bildumako liburuak » [Livres de la collection], <https://eizie.eus/eu/argitalpenak/literatura_unibertsala/orainarte $>$ [consulté le 14.05.2020].

33 « Amaia Apalauza Ollo », [dans :] Wikipedia. La enciclopedia libre, <https://eu.wikipedia. org/wiki/Amaia_Apalauza> [consulté le 14.05.2020]. 
parce qu'il reflète bien le regard particulier de l'auteur sur les événements dans le monde et en raison de sa façon exceptionnelle de les raconter ${ }^{34}$.

Les œuvres polonaises traduites en basque sont trop rares pour qu'on puisse en tirer des conclusions certaines, mais la présence de l'auteur et de son œuvre en castillan semble toutefois y être pour quelque chose. Kapuściński est l'un des rares auteurs polonais dont presque toutes les œuvres aient été traduites en espagnol. Panny z Wilka est paru la même année que son édition polonaise de Madrid. Le livre de Tokarczuk vient comme complément des éditions espagnole et catalane.

\section{AUTRES TRADUCTIONS GALICIENNES. MROŻEK ET SZYMBORSKA EN CATALOGNE}

Zgubiona dusza n'est pas non plus la première traduction d'œuvre de la littérature polonaise en langue galicienne. La première, en effet, aura été un roman d'Andrzej Zaniewski, Szczur, paru en 1995 sous le titre de rata $^{35}$. La situation de ce livre est particulière, car il a d'abord été publié à Paris, en traduction française, et ses versions allemande, néerlandaise et espagnole existaient déjà lorsqu'il est apparu dans les rayons des librairies polonaises. La base de données du MECD signale encore que la traduction a été réalisée à partir du polonais, ce que semble confirmer le nom de la traductrice ${ }^{36}$. A rata est donc paru parallèlement à l'édition polonaise.

L'une des 32 entrées du catalogue du MECD correspondant aux œuvres de Sławomir Mrożek a été éditée en galicien. Elle s'intitule O prezo da fama ${ }^{37}$ (Cena stawy), porte ainsi le titre d'une des nouvelles du recueil Małe prozy [Petites proses] publié en 1990, et ne correspond à aucune des 31 autres entrées de la base de données. Il s'agit peut-être d'une opération de l'éditeur visant à attirer l'attention de lecteurs qui, sur le marché, avaient depuis longtemps accès à un grand nombre de titres de cet auteur. L'éditeur responsable de cette publication, Rino-

34 N. Velez, « Amaia Apalauza: 'Lo más especial en la obra de Kapuscinski es su mirada'. Entrevista », Euskal Irrati Telebista, 07.12.2019, <https://www.eitb.eus/es/cultura/literatura/ detalle/6851370/entrevista-amaia-apalauza-traductora-kristo-fusilarekin-kapuscinski-/> [consulté le 14.05.2020].

35 A. Zaniewski, A rata, trad. M.C. Costas Fernández, Edicións Xerais de Galicia, Vigo 1995. La base de données de la Bibliothèque nationale indique que la traduction a été effectuée à partir du polonais, mais comme me l'a fait observer un des réviseurs anonymes du présent article, selon la base de données Bitraga (Biblioteca de tradución galega), qui présente les travaux de la traductrice, le roman a été traduit de l'anglais.

36 A. Zaniewski, La rata, trad. I. Ochlewska-Fernández, Alianza Editorial, Madrid 1994.

37 S. Mrożek, O prezo da fama, trad. P. Cancelas, Rinoceronte Editora, Cangas 2011. Selon la base de données de la Bibliothèque nationale, la traduction a été réalisée à partir de l'original. L'information est confirmée par un article consacré à la traductrice, que m'a signalé le réviseur anonyme : M.G., « Un puente entre Polonia y Moaña », Faro de Vigo, 23.05.2007, <https://www.farodevigo.es/o-morrazo/2007/05/23/puente-polonia-moana-18135678.html> [consulté le 8.03.2021]. 
ceronte Editora, a probablement tablé sur la rareté. Il n'a donc choisi aucun des titres déjà connus grâce à leurs versions castillane ou catalane, partant peut-être du principe que les amateurs de Mrożek ou de la littérature polonaise rechercheraient d'abord la version castillane, et que l'indisponibilité du livre dans cette langue les inciterait à le lire en galicien, langue que les autres Espagnols comprennent, surtout à l'écrit ${ }^{38}$.

La présence de Mrożek en Espagne remonte à 1963, et il n’y est pas arrivé via la France, mais via le monde germanique : ses deux premières traductions ont été réalisées à partir de l'anglais et de l'allemand. Après la traduction de Violetta Beck et Jorge Segovia de 1973 — la première à partir de l'original —, Mrożek a ensuite été oublié pendant 18 ans. Sa réapparition en Espagne est une histoire strictement catalane : à partir de 1991, il devient l'affaire des éditeurs de cette région (Seix Barral, Acantilado et Quaderns Crema de Barcelone, et Vicens Vives de Valence), qui le publient toutefois souvent en deux versions linguistiques parallèles. On voit alors paraître en catalan des titres de lui qui n'ont jamais été publiés en espagnol (Una nit qualsevol - Czarowna noc, L'escorxador $i$ altres peces - Rzeźnia $i$ inne sztuki), mais la situation inverse est vraie aussi (Feliz acontecimiento - Szczęśliwe wydarzenie). À partir de 1997, l'édition des livres de Mrożek entre dans une nouvelle phase : les éditeurs barcelonais — surtout Acantilado, dirigé par Jaume Vallcorba Plana - commencent à livrer à leurs lecteurs des versions parallèles en castillan et catalan. Avec le temps, cette rigueur d'édition va se relâcher, et les éditions en espagnol vont prendre le dessus, étant adressées à un public plus large. On peut toutefois considérer que pour ce qui est des traductions de Mrożek, le catalan n'a jamais été dominé par l'espagnol et a toujours été sur un pied d'égalité avec lui. Peut-être Rinoceronte Editora a-t-elle voulu profiter de l'expérience catalane et a-t-elle voulu essayer de promouvoir Mrożek sur son propre terrain, en Galice.

Les raisons de l'édition de la poésie de Wisława Szymborska en galicien, et plus généralement en Espagne ne font évidemment aucun doute, comme le prouve la date des premières éditions, 1997, c'est-à-dire l'année qui a suivi le prix Nobel de la poétesse. Avant de recevoir le Nobel, Szymborska était pratiquement inconnue en Espagne, ou en tout cas, ses œuvres n'y avaient jamais été éditées sous forme de livre. C'est la Catalogne qui a réagi le plus rapidement : la plupart des éditeurs espagnols de Szymborska sont basés dans cette région (à Barcelone, Tarragone et Vic). Mais c'est en castillan qu'elle est le plus traduite. Son édition galicienne est bilingue et n'a vu le jour que très tardivement, quinze ans après le Nobel. Son éditeur n'a pas non plus porté son choix sur l'un des recueils de poèmes existants et s'est contenté d'une anthologie (Versos escollidos ${ }^{39}$ signifie

38 Selon le réviseur anonyme, il est fort probable que Rinoceronte Editora ait choisi ce titre qui n'était pas encore traduit en espagnol pour éviter la concurrence qu'aurait représenté, en Galice, une version dans cette langue.

39 W. Szymborska, Versos escollidos, trad. L. Caeiro, Edicións Positivas, Santiago de Compostela 2011. 
« vers choisis »). Pour être complet et pour en terminer avec la situation du catalan, on peut ajouter qu'après l'édition galicienne, Szymborska a surtout été publiée en espagnol, mais que le catalan est peut-être en train de rattraper son retard, car un ouvrage de la poétesse est paru dans cette langue en $2018^{40}$.

La publication de la poésie de Wisława Szymborska et du roman d'Olga Tokarczuk n'a rien de surprenant : dans les deux cas, il s'agit d'œuvres consacrées par un prix littéraire international prestigieux. C'est probablement le même genre de motivation qui est intervenu dans le cas d'une autre œuvre polonaise traduite de l'anglais en galicien, Gottland de Mariusz Szczygiel ${ }^{41}$. En 2007, le livre a collectionné les prix polonais, en 2 ans, il a été traduit dans 6 langues, et en 2009, il a reçu le Prix du livre européen, autant d'arguments de poids. La même année, le Prix Amphi a été décerné à Szczygieł et à sa traductrice française Margot Carlier. De quoi encourager les Espagnols à le traduire. Mais en Galice plus particulièrement, Gottland a été sélectionné par la Xunta de Galicia et a reçu le financement « Traduction 2017 » du département de la culture ${ }^{42}$. Les innombrables langues dans lesquelles Gottland a été traduit avant sa parution en Galice (tchèque, français, allemand, italien, hongrois, russe, ukrainien, espagnol, slovène, slovaque, serbe, bulgare, anglais, estonien et roumain) ont certainement pesé dans la décision de la Xunta. Cependant, l'intervalle de temps entre la sortie de l'original et la traduction en galicien - 11 ans - semble être un signe évident de la nature périphérique de ce marché éditorial. À ce propos, il est intéressant de noter que les Catalans n'ont pas traduit Szczygieł dans leur propre langue, ce qui est assez surprenant étant donné qu'il a été publié par Acantilado, un éditeur catalan.

Face aux Tokarczuk - Szymborska - Szczygieł — Mrożek, la traduction suivante de littérature polonaise entreprise par les éditions Rinoceronte peut paraître un choix très atypique, puisqu'il s'agit de Mikołaja Doświadczyńskiego przypadki $^{43}$. En effet, contrairement aux livres évoqués jusqu'à présent, ce roman ne fait pas partie de la littérature contemporaine, c'est-à-dire de celle que l'on traduit habituellement. Il avait jusque là déjà été traduit en trois langues que $\mathrm{Ca}$ sanova appelle centrales : le français, l'allemand et l'anglais. L'édition anglaise est relativement récente (1992), mais malgré tout très antérieure à la version galicienne. En revanche, le roman n'a jamais été traduit en espagnol ni dans une autre langue de la Péninsule ibérique. C'est cette circonstance, semble-t-il, qui a motivé l'éditeur. Les informations publiées sur son site - qui présente l'éditeur et son

40 W. Szymborska, Instant, trad. J. Bielak, Eumo Editorial SAU, Barcelona 2018.

41 M. Szczygieł, Gottland, trad. M. Barcia, Rinoceronte Editora, Cangas 2018.

42 Diário Oficial da Galiza, Resolução do DOG no 106 do 2017/6/6 - Xunta de Galicia, $<$ https://www.xunta.gal/dog/Publicados/2017/20170606/AnuncioG0164-240517-0001_pt.html> [consulté le 14.05.2020]; Rinoceronte Editora, <https://rinoceronte.gal/editora/pf/gottland/> [consulté le 14.05.2020].

43 I. Krasicki, As aventuras de Mikołaj Doświadczyński, trad. L. García Sánchez, Rinoceronte Editora, Cangas 2019. 
catalogue d'auteurs - laissent entendre que Rinoceronte se spécialise dans les titres inconnus du lecteur espagnol et pour lesquels les grandes maisons d'édition n'ont qu'un intérêt marginal. Il publie de la littérature polonaise, mais aussi des œuvres traduites d'autres langues telles le russe, hongrois, japonais, nigérien, turc, finnois, norvégien, islandais, grec, ainsi que de la littérature italienne ancienne ${ }^{44}$.

Si l'on inclut dans la littérature polonaise non seulement les textes écrits en polonais, mais aussi ceux écrits par des Polonais - catégorie dans laquelle sont habituellement classés Joseph Conrad ou le Manuscrit trouvé à Saragosse -, une des œuvres publiées par Rinoceronte doit encore être signalée : les mémoires de Józef Boruwłaski, aristocrate polonais et nain de cour du XVIII e siècle qui fut le protégé de Stanislas-Auguste Poniatowski. Boruwłaski a voyagé dans toute l'Europe pour finalement s'installer en Angleterre, où il a publié ses mémoires en français et en anglais. Elles ont ensuite été traduites en allemand, puis rééditées en français et anglais quelques années plus tard. Elles n'ont été traduites en polonais qu'en 2004, par Anna Grześkowiak-Krwawicz. Une nouvelle édition française a vu le jour en 2008, et la traduction espagnole, en $2010^{45}$. Rinoceronte en a publié une version galicienne quatre ans plus $\operatorname{tard}^{46}$. Ce titre ne semble pas faire partie des habitudes de la maison, mais peut-être la version espagnole était-elle épuisée et l'éditeur y a-t-il vu une occasion?

\section{AUTRES TRADUCTIONS CATALANES}

Il résulte des données chiffrées évoquées ci-dessus qu'environ un douzième des traductions de littérature polonaise réalisées en Espagne sont des traductions en catalan. Le nombre d'éditions dans cette langue, supérieur à celui du basque et du galicien, permet de dresser un tableau de la littérature polonaise en catalan sans entrer dans le détail des titres et, cela va sans dire, d'observer certaines tendances que j'ai peut-être déjà réussi à identifier partiellement dans le cas des traductions de Mrożek. La première tendance, très nette, est à la publication en parallèle des traductions en catalan et en castillan. Cette politique éditoriale est évoquée par José Díaz dans la citation ci-dessus et se laisse observer en dressant un simple tableau des éditions de littérature polonaise année après année. À côté des nombreuses traductions de Mrożek, apparaissent un ensemble d'autres œuvres. Certaines d'entre elles ont d'abord eu une version espagnole, mais les éditions

44 Rinoceronte Editora, « Autores », <https://rinoceronte.gal/editora/autores-2/> [consulté le 14.05.2020] ; Rinoceronte Editora, « Quen somos », <https://rinoceronte.gal/editora/quen-somos/> [consulté le 14.05.2020].

45 J. Boruwlaski, Memorias del célebre enano Joseph Boruwlaski, gentilhomme polaco, trad. V. Fernández Camarero, Lengua de Trapo, Madrid 2010.

46 J. Boruwlaski, Memorias do famoso anano Joseph Boruwlaski, trad. R. Vizcaíno Bravo, Rinoceronte, Cangas 2014. 
catalane et castillane du même titre sont datées de la même année ${ }^{47}$. Le premier exemple de ce type est celui de Quo vadis de Henryk Sienkiewicz (1964). Comme l'indique Ilona Narębska, la version catalane a été réalisée après la castillane, mais elles ont été publiées en parallèle la même année, et de surcroît, il y en a eu quatre en espagnol cette année-là ${ }^{48}$.

La deuxième tendance, plus rare, consiste à rattraper le retard en traduisant une œuvre qui existe déjà en espagnol. C'est par exemple le cas du deuxième titre polonais à avoir été traduit en catalan : Ferdydurke de Witold Gombrowicz ${ }^{49}$. Mais même dans ce cas, on peut observer une analogie avec la première tendance : c'est bien le texte espagnol qui a servi de base au catalan ${ }^{50}$, mais ce dernier est paru en même temps que deux autres œuvres de Gombrowicz en castillan, Seducción et Ivonne, princesa de Borgoña ${ }^{51}$. Ces deux titres ont d'ailleurs été traduits en catalan les années suivantes par Dorota Szmidt (Pornografia et Yvonne, princesa de Borgonya sont parus en 1990). Et on pourrait citer d'autres exemples ${ }^{52}$.

47 Curs de filosofia en sis hores i quart / Curso de filosofia en seis horas y cuarto de W. Gombrowicz (1997), El manuscrit trobat a Saragossa / Manuscrito encontrado en Zaragoza de J. Potocki (1990), Informe des de la ciutat assetjada / Informe desde la ciudad sitiada y otros poemas de Z. Herbert (1993), L'escena de la memòria / La escena de la memoria de T. Kantor (1997), Vista amb un gra de sorra: antologia poetica / Paisaje con grano de arena de W. Szymborska (1997), La senyoreta Ningú / Niña nadie de T. Tryzna (1998), Solaris de S. Lem (2001), Un lloc anomenat Antany / Un lugar llamado Antaño de O. Tokarczuk (2001), Madame de A. Libera (2002), La bella senyora Seidenman / La bella señora Seidenman de A. Szczypiorski (2002), Un dia més de vida / Un día más con vida (2003, 2008, 2010), Viatges amb Heròdot /Viajes con Heródoto (2006), Crist amb la carrabina al coll / Cristo con un fusil al hombro (2010) de R. Kapuściński, El pianista del gueto de Varsòvia / El pianista de W. Szpilman (2003), Aixaqueu-vos, anem! / Levantaos! Vamos! (2004) et Estic a les teves mans: quaderns personals 1962-2003 / Estoy en tus manos: cuadernos personales, 1962-2003 (2014) de Jean Paul II, Terra del foc / Tierra del fuego de A. Zagajewski (2004), Benvinguts a Mamoko / Bienvenidos a Mamoko (2013) et El meu día / Mi día (2017) de A. et D. Mizieliński, El llenyater / El leñador de M. Witkowski (2013), El diari de la Rywka: La veritabla història d'una nena jueva al gueto de Lodz / El diario de Rywka Lipszyc de R. Lipszyc (2015), Kulinari kultiva / Kulinario kultiva de K. Bogucka et S. Tomilo (2016), Ecologic Kultiva / Ecológico kultiva de Baobaby Studio: A. Pakalska et E. Bielańska (2016), Mercuri a la boca / Tragar mercurio de W. Greg (2017), Muntanyes / A la montaña! de P. Karski (2017), Formes trapelles / Formas traviesas de A. Królak (2018), El fil d'Ariadna: mites i laberints / El hilo de Ariadna: mitos y laberintos de J. Bajtlik (2018), El gran circ Iris / El gran circo Iris de B. Pajdak (2019), Sonka de I. Karpowicz (2020).

48 I. Narębska, Literatura polaca en España (1939-1975): autores, editores, traductores, Wydawnictwo Wyższej Szkoły Filologicznej we Wrocławiu, Wrocław 2015, pp. 67-68.

49 Ibidem, pp. 71, 304.

50 Qui d'ailleurs n'a pas été édité en Espagne ni a cette époque : W. Gombrowicz, Ferdydurke, trad. W. Gombrowicz, Argos, Buenos Aires 1947.

51 I. Narębska, op. cit., p. 71.

52 La mare: comèdia de mal gust en dos actes i epíleg de S.I. Witkiewicz (1987, éd. espagnole 1973), Cosmos (1998, éd. espagnole 1969) et Diari (1953-1956) de W. Gombrowicz (1999, éd. espagnole 1993), La vall de l'Issa (1998, éd. espagnole 1981) et La ment captiva (2005, éd. espagnole 1981) de C. Miłosz, L'enamorat de l'Óssa Major de S. Piasecki (2001, éd. espagnole 1944), Les botigues de color cangella; El sanatori de la clepsidra de B. Schulz (2001, éd. espagnoles 
La troisième situation, plus rare encore dans le cas du catalan, est celle des livres qui n'ont pas été d'abord édités en castillan. Outre les œuvres de Mrożek déjà évoquées, on y trouve : El pop o La visió hyrkanesa del món, Sobre la forma pura de Stanisław Ignacy Witkiewicz (1992), Angoixa (2002) et Udols d'un llop de paper (2010) de Tadeusz Różewicz, Eixida d'emergència d'Ewa Lipska (2004), Un bárbar al jardí de Zbigniew Herbert (2009), Amb els ulls d'una nena de dotze anys de Janina Hescheles-Altman (2012), El defecte de Magdalena Tulli (2015), Una dona de cap a peus (2015), El llapis amb què escric (2019) d'Anna Świrszczyńska et No vull ser princesa! de Grzegorz Kasdepke (2019).

\section{CONCLUSION}

Ce passage en revue des livres publiés en Espagne dans d'autres langues que le castillan permet de tirer certaines conclusions. Le caractère périphérique d'une langue peut être mesuré par le nombre de traductions produites dans cette langue, mais aussi par le temps écoulé entre la publication de l'original et l'édition en langue cible. Plus ce temps est long, plus la langue est éloignée du centre. En Espagne, pour ce qui est de la littérature polonaise, c'est la langue basque (je ne tiens plus compte ici du léonais, de l'aragonais et de l'aranais) qui se situe dans la périphérie la plus éloignée, avec seulement trois traductions dont deux réalisées plusieurs décennies après les originaux.

Les prix littéraires internationaux semblent être un critère important pour les traductions en langues périphériques : le prix Nobel a motivé l'édition de Zgubiona dusza dans deux langues de plus que prévu et a fait connaître Szymborska en Espagne, et le Prix du livre européen a fait la promotion de Gottland. D'autre part, les prix sont généralement attribués à des œuvres qui ont déjà été traduites dans plusieurs langues.

Dans les communautés multilingues, la traduction vers les langues dominées suivent l'un des deux modèles suivants, que j'appellerai pour la convenance : 1) le catalan et 2) le galicien. Pour parler par métaphore, ces langues ont le choix entre 1) un combat inégal pour le rang et le prestige de leur littérature traduite en s'alignant sur le rythme imposé par leur rivale (éditions ou rééditions simultanées), ou 2) accepter leur caractère périphérique et rechercher un créneau que la langue dominante semble ignorer, en éditant principalement des titres et des auteurs inconnus dans celle-ci. Bien sûr, la situation du catalan et du galicien telle que je viens de l'évoquer montre qu'il ne faut pas s'attendre à ce que ces modèles se présentent sous une forme pure. Ils sont plutôt à comprendre comme une cer-

1972 et 1986), Pensaments despentinats de S.J. Lec (2003, éd. espagnole 1997), Una missa per la ciutat d'Arràs de A. Szczypiorski (2004, éd. espagnole 1991), Eben de R. Kapuściński (2006, éd. espagnole 2002), El diari de la Renia: L'Holocaust a través dels ulls d'una adolescent de R. Spiegel (2020, éd. espagnole 2019). 
taine tendance dominante. En effet, on trouve sur le marché espagnol des titres traduits en catalan qui n'ont pas encore paru en version castillane, alors qu'en Galice, c'est plutôt la situation inverse qui peut être observée ${ }^{53}$. La présence d'une édition espagnole semble très importante dans le cas de la littérature traduite en basque : les rares traductions dans cette langue doublent des versions espagnoles existantes, et parfois même, sont réalisées à partir de celles-ci. Pour répondre à la question initiale, on peut dire que dans les communautés multilingues, selon le modèle adopté, la présence d'une traduction dans la langue dominante peut être à la fois une motivation et un frein aux traductions en langues minoritaires, mais que dans tous les cas, elle reste toujours un point de référence majeur.

Traduit par Xavier Chantry

\title{
TRANSFER OF FOREIGN LITERATURE IN BILINGUAL CONDITIONS: POLISH LITERATURE IN THE PERIPHERAL LANGUAGES OF SPAIN AND ITS EARLIER CASTILIAN TRANSLATIONS
}

\begin{abstract}
The study tries to answer the question of how literary translation functions in bilingual conditions. By analysing the history of Polish literature in Spanish, Catalan, Galician and Basque, the author attempts to explain how the translation into a dominant language affects translations in dominated languages. The results of the study indicate that the dominant language is always a reference point for translations in peripheral languages, although it can be both an impulse and an obstacle to their creation depending on the model of conduct adopted by the language.
\end{abstract} transfer.

Key words: Polish literature, translation, Basque, Galician, Catalan, bilingualism, literature

${ }^{53}$ La situation du catalan est nettement différente de celle du galicien et du basque : il lui est plus facile de faire concurrence au castillan car il dispose d'un lectorat fidèle et l'importance de l'industrie du livre catalane lui permet de publier non seulement dans toute l'Espagne, mais aussi dans les deux Amériques. Le galicien et le basque n'ont pas ces possibilités, même si leur dépendance du castillan a diminué ces dernières années.

J'adresse mes vifs remerciements au réviseur anonyme de cet article pour cette dernière remarque comme pour les précédentes et pour m'avoir signalé l'existence de bases de données de livres publiés en basque, galicien et catalan. 